\title{
Creation of a Virtual Nutrition Support Team to Improve Quality of Care for Patients Receiving Parenteral Nutrition in a Multisite Healthcare System
}

\author{
Michelle Meyer PharmD, BCPS, BCNSP ${ }^{1}$, Jennifer Hartwell MD², Amy Beatty PharmD, \\ BCPS $^{3}$, Theresa Cattell RD, CNSC ${ }^{4}$
}

1. Grant Medical Center, OhioHealth Columbus, Columbus, Ohio, USA.

2. Grant Medical Center, Indiana University School of Medicine/Methodist Hospital, OhioHealth Columbus, Indianapolis, Indiana, USA.

3. OhioHealth Columbus, Columbus, Ohio, USA.

4. Riverside Methodist Hospital, OhioHealth Columbus, Columbus, Ohio, USA.

\begin{abstract}
BACKGROUND

Parenteral nutrition (PN) is a complicated therapy in which having specially trained clinicians can provide benefit, but it can be difficult to provide this level of expertise to all patients requiring PN. Creation of a virtual nutrition support team (NST) model allows patients across multiple hospitals to receive care from NST clinicians using remote conferencing technology on a daily basis. This study reviewed retrospective data from before and after implementation of the virtual model to assess quality indicators.
\end{abstract}

METHODS

The NST was developed including a team of dietitians and pharmacists with a physician medical director. Practice guidelines were developed to provide consistent methods for ordering and monitoring patients receiving PN. Patient charts in both the preintervention and postintervention groups were reviewed for indication for PN, duration of therapy, blood glucose levels, and demographic data.

RESULTS

A greater proportion of patients in the postintervention period had appropriate orders $(97.2 \%)$ compared with patients in the preintervention period $(58.9 \%)(P<0.001)$. A greater proportion of patients in the postintervention period had blood glucose levels within the range $65-180 \mathrm{mg} / \mathrm{dL}(83.5 \%)$ compared with patients in the preintervention period $(62.2 \%)(P<0.001)$.

CONCLUSION

A virtual team model was applied to remotely manage patients receiving PN in a large healthcare system. This resulted in optimized care of patients by reducing inappropriately prescribed therapy and improving blood glucose control.

Keywords: nutrition support, nutrition support team, parenteral nutrition, telemedicine, videoconferencing.

This is the author's manuscript of the article published in final edited form as:

Meyer, M., Hartwell, J., Beatty, A., \& Cattell, T. (2019). Creation of a Virtual Nutrition Support Team to Improve Quality of Care for Patients Receiving Parenteral Nutrition in a Multisite Healthcare System. Nutrition in Clinical Practice, 34(6), 881-886.

https://doi.org/10.1002/ncp.10375 


\section{BACKGROUND}

Parenteral nutrition (PN) is a specialized form of nutrition support. The complications associated with the administration of PN can be classified as mechanical, infectious, metabolic, and nutritional. 1 , 2 The Institute for Safe Medication Practices has given PN preparations the status of a high-alert medication because of increased risk of harm if used in error. 3 Because of the complexity of PN and associated complications, a high level of knowledge and expertise is recommended in the management of these patients.4 Physicians receive minimal training and experience in the management of PN in standard education. $\underline{\mathbf{5}}, \underline{6}$ The American Society for Parenteral and Enteral Nutrition (ASPEN) recommended in 2010 Standards for Nutrition Support that patients receiving PN have a nutrition support team (NST) managing the care of the patient.7 An NST is made up of clinicians with specialty training in nutrition and can include physicians, dietitians, pharmacists, and nurses. $\underline{8}$ The multidisciplinary team approach has been supported for its ability to assess a patient's nutrition status, prescribe the appropriate macronutrients and micronutrients, and decrease the incidence of metabolic complications through regular monitoring. $\underline{\underline{g}}, \underline{9} \mathrm{PN}$ is a more expensive form of nutrition support compared with enteral nutrition (EN), and the multidisciplinary NST can assess for the appropriateness of PN and the timely transition to enteral or oral nutrition when indicated, resulting in cost savings. $\underline{4}, \underline{10}-\underline{12}$

Despite the demonstrated benefits associated with an NST, it can be difficult for a small institution to have the resources available for this service. The volume of patients receiving $\mathrm{PN}$ may be too low to rationalize specialized training by clinicians. Even at a larger institution, there are challenges in maintaining clinician competency in PN because of fluctuations in patient volumes, competing patient care priorities, and planned clinician absence. Using a virtual NST to care for patients at multiple sites addresses both of these concerns by allowing sites with smaller PN volumes to share resources with larger sites and justifies the time required for clinicians to maintain specialized training in nutrition support.

Our system of care sites includes 10 hospitals in the Midwestern United States. Hospitals range from 25 beds to 1059 beds. The hospitals included in the evaluation are shown in 
Table 1. Historically, PN ordering and practices varied at each site. Some sites had a nutrition support clinician evaluating patients daily and others only 5 days per week. Most adult patients had new orders placed only for a change in formula. In some sites, glucose control was managed by a nutrition support clinician, but at other sites, the primary team managed blood glucose.

Table 1. System Hospitals Descriptions ${ }^{\underline{a}}$

\begin{tabular}{|l|l|l|l|}
\hline Hospital & Hospital Type & $\begin{array}{l}\text { Registered } \\
\text { Beds }\end{array}$ & $\begin{array}{l}\text { Pre-Implementation PN Orders Generally } \\
\text { Recommended By }\end{array}$ \\
\hline 1 & $\begin{array}{l}\text { Community } \\
\text { teaching }\end{array}$ & 262 & Unit dietitian \\
\hline 2 & Community & 107 & Unit dietitian/pharmacist \\
\hline 3 & $\begin{array}{l}\text { Community } \\
\text { center 1 trauma }\end{array}$ & 636 & Unit dietitian \\
\hline 5 & $\begin{array}{l}\text { Tertiary care, } \\
\text { teaching }\end{array}$ & 1059 & Unit dietitian \\
\hline
\end{tabular}

- a There are 10 hospitals in the system, but only these 5 were included in the evaluation.PN, parenteral nutrition.

With the institution of a new electronic health record (EHR), an opportunity for standardization was created. It was proposed that patients requiring PN could be managed virtually for all care sites by a small group of clinicians, allowing a higher level of expertise in nutrition support. By implementing a virtual NST, we expected to standardize the care and monitoring of patients receiving $P N$, including daily review in accordance with ASPEN recommendations. $\underline{7}, \underline{13}$ It was hypothesized that this standardized approach would reduce the frequency of inappropriate PN orders as well as result in improved glucose control for patients receiving PN. 


\section{METHODS}

\section{IMPLEMENTATION OF THE VIRTUAL NST}

In light of the identified system-wide gaps in PN ordering and monitoring, beginning in 2015, a virtual NST model was proposed, developed, and implemented across hospitals. The system-wide transition to a new EHR platform facilitated the coordinated EHR build and launch of this virtual model across all hospitals. The service was socialized and deployed systematically upon each site's go-live on the new EHR platform over a period of 9 months. This allowed the virtual team to make adjustments to manage the workflow and increasing volumes over the 9-month rolling go-live. At the time of the postintervention evaluation, 5 sites were included in the data.

First, the team identified a multidisciplinary team of registered dietitians and pharmacists with a physician medical director. Competency was assessed by using ASPEN education module completion and case studies evaluated by the NST medical director for all team members, using board-certified clinicians as the core of the team.

Second, to standardize care within the team, practice guidelines were developed to provide clinicians with consistent methods for carrying out patient care activities related to ordering and providing PN. A Pharmacy and Therapeutics policy was developed to dictate that all adult PN orders, including orders for critical care, intermediate care, and general medical-surgical care patients, must be completed by the NST and transmitted directly to the pharmacy. Specifically, patients receiving PN are followed daily by a specialty trained dietitian and pharmacist who are responsible for metabolic monitoring, daily PN prescribing, and assessment of continued clinical indication for the therapy.

An initial nutrition assessment is performed to determine calorie, protein, and micronutrient needs, as well as baseline glycemic control and existing or plan for intravenous access. Communication between the virtual NST and unit clinicians was maintained to ensure accurate information, assist with physical exam if needed, and enhance continuity of care. Regular communication with unit clinicians is essential in a virtual model, as bedside documentation can be limited by inaccuracies in reporting intake and output, meal consumption, and other charting. Methods included verbal 
communication, EHR charting, and communication via EHR tools (eg, handoff notes) at a frequency of at least daily based on the patient's acuity level and stability on the PN formula. Each day, patients with PN orders are assessed for appropriateness for PN based on 2016 ASPEN guidelines for critically ill patients and 2010 guidelines and ASPEN consensus statement for noncritically ill patients. $\underline{7}, \underline{14}, \underline{15}$

The NST also manages electrolyte and acid-base disorders related to PN in consultation with the primary team as needed. Glucose management is generally achieved with provision of insulin within the PN, although subcutaneous basal and corrective insulin and insulin infusions may be comanaged with the medical provider or endocrinologist. Based on the ASPEN recommendation to monitor and reorder daily on many patients, the decision was made to reorder all PN orders daily.13, $16 \mathrm{~A}$ visual representation of the process workflow for ordering PN can be seen in Figure 1. NST members monitor for continued clinical indication and progress toward transition to EN and/or oral diet while maintaining communication with unit clinicians. Remote management is facilitated by a daily conference between the NST dietitian and the NST pharmacist to review patients and develop individualized care plans.
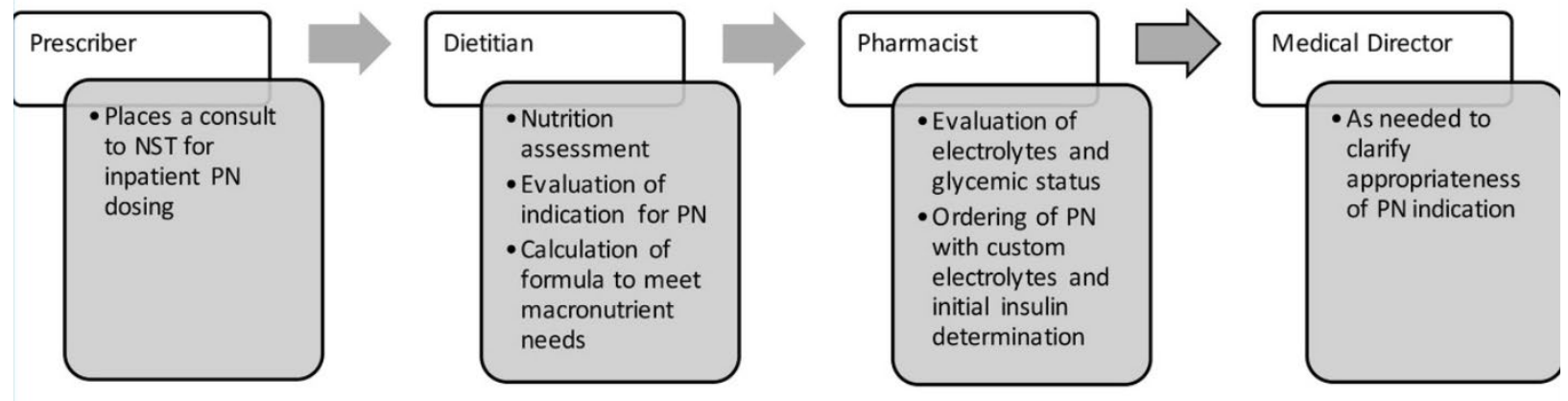

Figure 1: Virtual NST workflow. NST, nutrition support team; PN, parenteral nutrition.

\section{EVALUATION OF THE VIRTUAL NST}

Three areas were identified prior to implementation to measure satisfactory function of the virtual NST: increasing the frequency of patient evaluation to daily on all patients, 
maintenance of adequate blood glucose control, and restricting PN to patients with defined appropriate indication.

Success of the virtual NST was evaluated by comparing retrospective data for all adult patients ( $\geq 18$ years of age) who were prescribed $P N$ at any of the 5 system hospitals during a 6-month period prior to implementation of the NST (July-December 2013), and a 6-month period after the NST was implemented across all 5 target hospital sites (JuneNovember 2015). Approval for retrospective chart review was granted by the system's Institutional Review Board. Data collected included baseline PN orders across the included hospital sites (including total days of PN), blood glucose measurements while receiving $\mathrm{PN}$, and indications for parenteral therapy.

\section{STATISTICAL ANALYSIS}

Demographic characteristics of patients $\geq 18$ years of age who were prescribed PN during an inpatient hospital stay across 5 hospitals during the preintervention and postintervention periods were described using frequencies and percentages for categorical variables and means and SD for continuous variables. Demographic characteristics of patients seen during the preintervention and postintervention periods were compared using $X^{2}$ tests for categorical variables and 2-sample $t$-tests for continuous variables. Statistical significance was set at $P<0.05$ for all tests. PN orders,

including total days of $\mathrm{PN}$ and indications for $\mathrm{PN}$, were described using frequencies and percentages for categorical variables and medians and ranges for continuous variables, overall and stratified by preintervention and postintervention. PN orders were compared between the preintervention and postintervention periods using $x^{2}$ and Wilcoxon rank sum tests. The proportion of patients with blood glucose levels within the range 65$180 \mathrm{mg} / \mathrm{dL}$ during the preintervention and postintervention periods was calculated and compared using a $x^{2}$ test.

\section{RESULTS}

A total of 420 patients were prescribed PN during the evaluation period (202 prior to implementation of the virtual NST and 218 following implementation of the NST). 
Demographics and clinical characteristics of the patients prescribed PN during the preintervention and postintervention intervals are described in Table $\underline{2}$.

Table 2. Demographics and Clinical Characteristics

\begin{tabular}{|l|l|l|l|l|}
\hline $\begin{array}{l}\text { Demographic and } \\
\text { Clinical } \\
\text { Characteristics }\end{array}$ & $\begin{array}{l}\text { Total } \\
(\mathbf{n = 4 2 0})\end{array}$ & $\begin{array}{l}\text { Preintervention } \\
(\mathbf{n = 2 0 2})\end{array}$ & $\begin{array}{l}\text { Postintervention } \\
(\mathbf{n = 2 1 8})\end{array}$ & $\begin{array}{l}\boldsymbol{P} \text { - } \\
\text { Value }\end{array}$ \\
\hline Female, n (\%) & $215(51.2)$ & $107(53)$ & $108(49.5)$ & 0.482 \\
\hline Age, mean (SD) & $\begin{array}{l}58.7 \\
(16.1)\end{array}$ & $58.7(17.1)$ & $58.8(15.1)$ & 0.941 \\
\hline \begin{tabular}{l} 
Indication for parenteral nutrition, $\mathrm{n}(\%)$ \\
\hline \multicolumn{1}{|c|}{ bowel } \\
obstruction
\end{tabular} & $157(37.4)$ & $65(32.2)$ & $92(42.2)$ & $<0.001$ \\
\hline Other & $134(31.9)$ & $91(45)$ & $43(19.7)$ & $<0.001$ \\
\hline Gastrointestinal fistula & $48(11.4)$ & $25(12.4)$ & $23(10.6)$ & 0.646 \\
\hline Failed enteral trial & $37(8.8)$ & $7(3.5)$ & $30(13.8)$ & $<0.001$ \\
\hline Short bowel syndrome & $28(6.7)$ & $7(3.5)$ & $21(9.6)$ & 0.017 \\
\hline Pancreatitis & $16(3.8)$ & $7(3.5)$ & $9(4.1)$ & 0.802 \\
\hline $\begin{array}{l}\text { Total days of parenteral } \\
\text { nutrition, median (range) }\end{array}$ & $6(1-49)$ & $6(1-34)$ & $7(1-49)$ & 0.589 \\
\hline
\end{tabular}

Overall, the most common indications for parenteral use were ileus/small bowel obstruction $(37.4 \%)$, other $(31.9 \%)$, and gastrointestinal fistula $(11.4 \%)$. Comparing before and after the creation of the virtual NST, "other" occurred significantly less often in the postintervention data, $19.7 \%$ compared with $45 \% \quad(P<0.001)$. Indications categorized as "other" included cases generally identified as appropriate (patient with 
bowel in discontinuity, chylothorax, preoperative nutrition support prior to major upper gastrointestinal surgery, etc), as well as patients in whom an indication could not be identified. The patients in whom the indication could not be identified mostly occurred in the preintervention group, in which the chart was being reviewed retrospectively, and standardized nutrition support documentation was not utilized at the time. When comparing the indication for PN to the ASPEN guidelines for critically ill and hospitalized patients, prescribing was categorized as either appropriate or inappropriate. $\underline{7}, \underline{14}$ If the indication was unable to be determined based on documentation, the indication was categorized as inappropriate. Some additional examples of patients with indications classified as inappropriate include poor oral intake without contraindication to enteral feeds and patients with no evidence of preexisting malnutrition started receiving PN prior 7 days without adequate intake. As shown in Figure 2 , a greater proportion of patients in the postintervention period had appropriate orders (97.2\%) compared with patients in the preintervention period $(58.9 \%)\left(X^{2}[1]\right.$ : $\left.92.27 ; P<0.001\right)$.

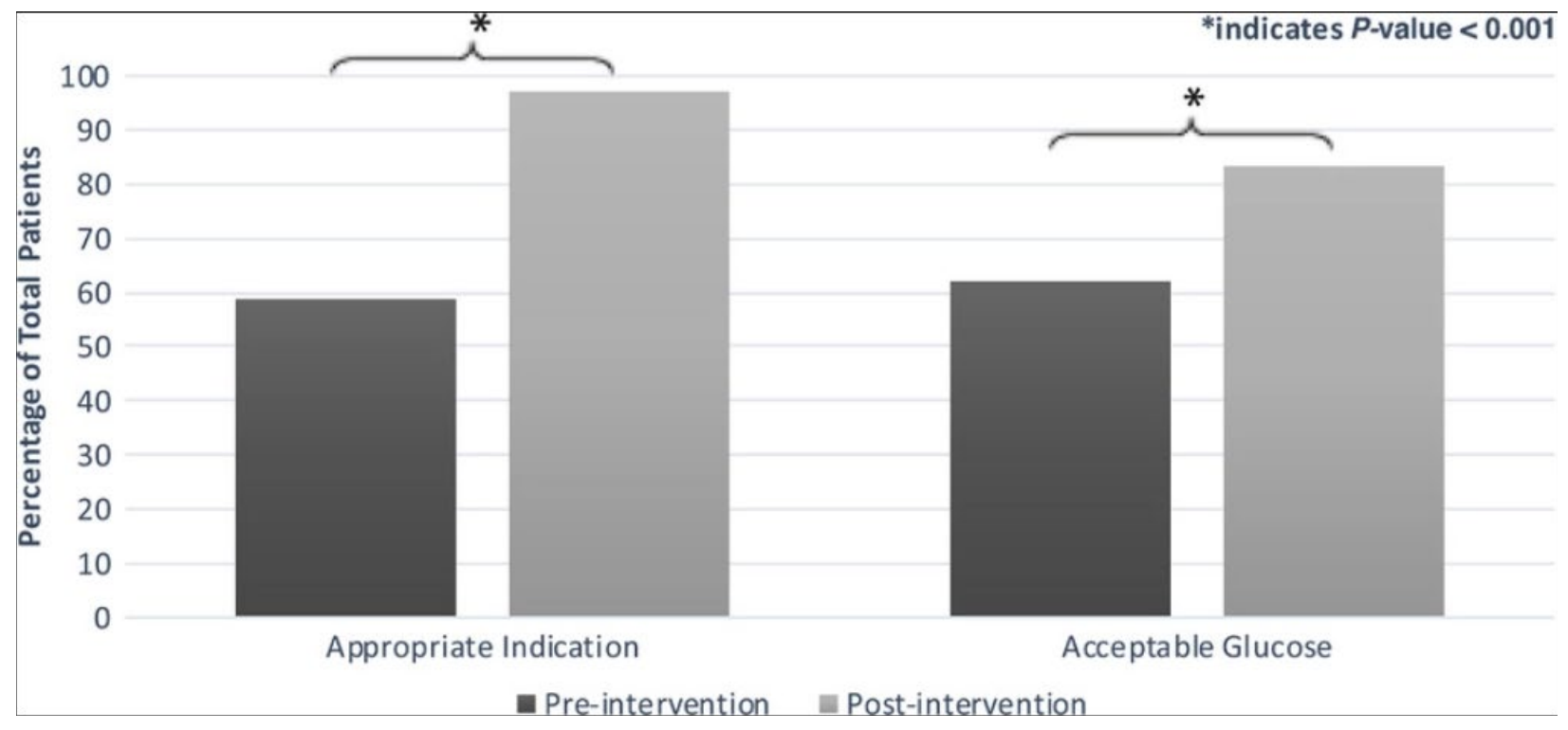

Figure 2: Quantity outcomes.

In comparing glucose management before and after implementing the virtual NST, blood glucose levels were based on recommendations from the NICE-SUGAR trial, endorsed by ASPEN. 17, 18 Goal blood glucose was defined as $65-180 \mathrm{mg} / \mathrm{dL}$. Standard protocol during administration of $\mathrm{PN}$ is to have glucose checked at bedside every 4 hours. 
Understanding that exceptions can occur, maintaining $\geq 80 \%$ of a patient's blood glucose readings in the defined range was considered to be acceptable glucose control. As shown in Figure 2, a greater proportion of patients in the postintervention period had blood glucose levels within the $65-180 \mathrm{mg} / \mathrm{dL}$ range $(83.5 \%)$ compared with patients in the preintervention period $(62.2 \%)\left(X^{2}[1]: 24.22 ; P<0.001\right)$.

\section{DISCUSSION}

The overarching goal of implementing the NST on a virtual level was to provide a higher level of expertise to our patients requiring PN without significantly changing resources across the system. There was a significant improvement in the number of patients with a PN indication that was able to be classified as appropriate after the implementation of the virtual NST. Some of this benefit we attributed to the careful evaluation of the NST clinician and discussion with the prescribers about possible opportunities for alternate nutrition. We do acknowledge that some increase in appropriateness can be attributed to poor documentation of indication in the preintervention group, especially as indications were classified as inappropriate if unable to be identified.

There were statistically significant increases in the percentage of indications for ileus/small bowel obstruction, failed enteral trial, and short bowel syndrome. Although this retrospective chart review cannot definitely determine reasons why the percentage of patients categorized as small bowel obstruction/ileus increased in the postintervention period, we suspect this is due to more comprehensive documentation by the ordering clinician after education from the NST about appropriate indications. An interesting finding is the statistically significant increase of the indication for PN being a "failed enteral trial" $(P<0.001)$. Although this retrospective review cannot provide all of the underlying reasons for this finding, we postulate that accountability for the initiation and maintenance of $\mathrm{PN}$, and daily encouragement to attempt enteral trials, may have prompted the treating physician to make more concerted efforts toward EN. Of those who had EN attempted, a portion will have succeeded and would have spared the need for PN. Though this retrospective review cannot address all of the added benefits of this finding, we presume, as has been demonstrated in a substantial body of literature and recommended by ASPEN, that EN is superior to PN whenever possible and results in improved outcomes, 
decreased morbidity, and decreased overall costs.10, $\underline{19}, \underline{20}$ As there was a substantial increase in the indication of "failed enteral trial," this shows evidence of a culture change in our health system toward increasing compliance in regard to nutrition best practices, that is, attempting EN prior to the use of PN.

By identifying the management of hyperglycemia as the scope of the NST, daily evaluation of the blood glucose and adjustment of insulin if needed became more standardized than the preintervention patients. The NST established guidelines for empiric insulin dosing and for increasing insulin in the PN bag based on amounts of corrective insulin received. We chose being within goal at least $80 \%$ of the time as the goal arbitrarily, acknowledging that excursions from the goal range may occur as we titrate the nutrition to goal. Even though the goal was determined without established standards defining consistency, the improvement from preintervention to postintervention is significant.

One of our goals of instituting the virtual NST was to standardize care across the system and an increase in patients being evaluated by a nutrition support-trained clinician daily when compared with PN. Prior to initiation of the virtual model, there was inconsistent monitoring and care of the patients. Some sites had a clinician evaluating patients daily and others only 5 days per week. Most adult patients had new orders placed only for a change in formula. In some sites, glucose control was managed by a nutrition support clinician, but at other sites, the primary team managed blood glucose. Following the institution of the virtual NST, every patient receiving PN at all sites was evaluated daily by a nutrition support dietitian and a pharmacist. We were unable to statistically evaluate the change in days of coverage because of limited availability of preintervention data in a retrospective review.

The original goal of remaining full-time equivalent (FTE) neutral was not met, and after initiation, there was a net expansion of 1 dietitian FTE and 0.4 pharmacist FTE. Although the NST model required increased FTE, the cost may be offset by decreased utilization of $\mathrm{PN}$, reduction of errors and complications, and improved care, which has been documented in patients managed by an NST.21 Although the cost analysis could not be 
completed in our retrospective review, we believe this is an important future area of study in our increasingly value-based healthcare system.

An added benefit of the virtual NST has been management of many medication shortages involving PN ingredients. The small number of clinicians allows easier dissemination and notification of shortages, updates to supply, and shortage management strategies. As all PN orders were written by the NST clinicians, it was also easier to maintain compliance with shortage management strategies.

Our work demonstrates the effectiveness of a virtual NST to provide superior level expertise to patients throughout a hospital system by improving outcomes, such as appropriately indicated and monitored PN administration and improved blood glucose levels, known to be associated with improved overall outcomes in hospitalized patients.22 This model allows an increased level of knowledge in the clinicians managing the PN, both by increasing the number of patients managed each month by the clinicians and reducing the number of clinicians responsible for managing PN. This also allows a smaller institution that may not be able to justify a certified nutrition support clinician to pair with other sites so that all patients receive a higher level of care.

Our next steps include expanding the quality assurance metrics we collect, further evaluating PN that is received for 5 days or less, and quantifying cost savings from decreasing inappropriate prescribing of PN. The latter could help justify the increase in FTE.

We recognize several limitations to our study. Although the virtual NST model was found to be an efficient and useful method for our mature 10-hospital system, the model may not be easily translatable to other systems that are either much larger or much smaller than the one we describe. However, the opportunity may exist for smaller entities to team up and centralize nutrition support expertise to improve care with limited expansion of resources. As with all retrospective studies, we relied heavily on the documentation provided in the daily progress notes and bedside documentation, which can sometimes be lacking or inadequate. At the time of data collection, we did not include an assessment on the acuity of the patients, as some patients were not in critical care and we were unable to calculate an Acute Physiologic Assessment and Chronic Health Evaluation or Charlson 
Comorbidity Index score to assess this. We would note that the service offerings within our hospitals (trauma, surgery, medical intensive care, etc.) did not change from the preintervention to the postintervention period.

In conclusion, a virtual team model was applied to remotely manage patients receiving $\mathrm{PN}$ in a large healthcare system. This resulted in optimized care of patients by reducing inappropriately prescribed therapy and improving blood glucose control.

\section{ACKNOWLEDGMENTS}

Statistical review for this article has been done by Christy Collins, $\mathrm{PhD}$, OhioHealth Columbus, Ohio.

\section{STATEMENT OF AUTHORSHIP}

M. Meyer, A. Beatty, J. Hartwell, and T. Cattell contributed to the conception/design of the research; M. Meyer and T. Cattell contributed to the acquisition, analysis, and interpretation of research; J. Hartwell and A. Beatty contributed to the analysis and interpretation of the data; and M. Meyer, J. Hartwell, and A. Beatty drafted the manuscript. All authors critically revised the manuscript, gave final approval, and agree to be accountable for all aspects of work, ensuring integrity and accuracy. 


\section{REFERENCES}

1. Btaiche, I. F., \& Khalidi, N. (2004). Metabolic complications of parenteral nutrition in adults, part 1. American Journal of Health-System Pharmacy, 61(18), 1938-1949. https://doi.org/10.1093/ajhp/61.18.1938

2. Btaiche IF, Khalidi N. Metabolic complications of parenteral nutrition in adults, part 2. Am J Health System Pharm. 2004; 61(19): 2050- 2057.

3. Insitute of Safe Medication Practices. ISMP List of high-alert medications in acute care settings. https://www.ismp.org/sites/default/files/attachments/201801/highalertmedications\%281\%29.pdf Accessed April 17, 2018.

4. Boullata J. Overview of the parenteral nutrition use process. JPEN J Parenter Enteral Nutr. 2012; 36(suppl 2): 10S-13S.

5. Adams K, Kohlmeier M, Powell M, Zeisel SH. Nutrition in medicine: nutrition education for medical students and residents. Nutr Clin Pract. 2010; 25(5): 471- 480.

6. Laszlo K, McClave S, Neel D, Evans D, Martindale R, Hurt R. Physician nutrition education. Nutr Clin Pract. 2014; 29(3): 332- 337.

7. Ukleja A, Freeman K, Gilbert K, et al. Standards for nutrition support: adult hospitalized patients. Nutr Clin Pract. 2010; 25(4): 403- 414.

8. Schneider P. Nutrition support teams: an evidence-based practice. Nutr Clin Pract. 2006; 21(1): 62- 67.

9. Naylor CJ, Griffiths R, Fernandez R. Does a multidisciplinary total parenteral nutrition team improve patient outcomes? A systematic review. JPEN J Parenter Enteral Nutr. 2004; 28(4): 251- 258.

10. Harvey SE, Parrott F, Harrison DA, et al. Intravenous nutrition is more costly than feeding by stomach tube, with few added benefits. Health Technol Assess. 2016; 20(28): 1- 144.

11. Parent B, Shelton M, Nordlund M, Aarabi S, O'Keefe G. Parenteral nutrition utilization after implementation of multidisciplinary nutrition support team oversight: a prospective cohort study. JPEN J Parenter Enteral Nutr. 2016; 40(8): 1151- 1157.

12. Giancarelli A, Davanos E. Evaluation of nutrition support pharmacist interventions. JPEN J Parenter Enteral Nutr. 2015; 39(4): 476- 481. 
13. Boullata J, Gilbert K, Sacks G, et al. A.S.P.E.N. clinical guidelines: parenteral nutrition ordering, order review, compounding, labeling, and dispensing. JPEN J Parenter Enteral Nutr. 2014; 38(3): 334- 377.

14. McClave S, Taylor B, et al. Guidelines for the provision and assessment of nutrition support therapy in the adult critically ill patient: society of critical care medicine (SCCM and american society for parenteral and enteral nutrition (A.S.P.E.N.). JPEN J Parenter Enteral Nutr. 2016; 40(2): 159- 211.

15. Worthington $P$, Balint J, Bechtold $M$, et al. When is parenteral nutrition appropriate? JPEN J Parenter Enteral Nutr. 2017; 41(3): 324- 377.

16. Ayers P, Adams S, Boullata J, et al; American Society for Parenteral and Enteral Nutrition. A.S.P.E.N. Parenteral nutrition safety consensus recommendations. JPEN J Parenter Enteral Nutr. 2014; 38(3): 296- 333.

17. NICE-SUGAR Study Investigators, Finfer S, Chittock D, et al. Intensive versus conventional glucose control in critically ill patients. $N$ Engl $J$ Med. 2009; 360(13): 1283- 1297.

18. McMahon M, Nystrom E, Braunscheig C. A.S.P.E.N. clinical guidelines: nutrition support of adult patients with hyperglycemia. JPEN J Parenter Enteral Nutr. 2013, 37;(1): 23- 36.

19. Seres D, Valcarcel M, Guillaume A. Advantages of Enteral nutrition over parenteral nutrition. Ther Adv Gastroenterol. 2013; 6(2): 157- 167.

20. Gramlich L, Kichian K, Pinilla J, Rodych N, Dhaliwal R, Heyland D. Does enteral nutrition compared to parenteral nutrition result in better outcomes in critically ill adult patients? a systemic review of literature. Nutrition. 2004; 20(10): 843- 848.

21.A.S.P.E.N. Practice Management Taskforce, DeLegge M, Wooley J, Guenter, P, et al. The State of nutrition support teams and update on current models for providing nutrition support therapy to patients. Nutr Clin Pract. 2010; 25(1): 76- 84.

22. Clement S, Braithwaite S, Magee M, et al. Management of diabetes and hyperglycemia in hospitals. Diabetes Care. 2004; 27(2): 553- 591. 
